\title{
Optimization of a Density Gradient Centrifugation Protocol for Isolation of Peripheral Blood Mononuclear Cells
}

\author{
Georgiana Mihaela Șerban, Ion Bogdan Mănescu, Doina Ramona Manu*, Minodora Dobreanu \\ University of Medicine and Pharmacy of Tîrgu Mureș, Romania
}

\begin{abstract}
Objective: Peripheral blood mononuclear cells (PBMC) are extremely important in the body's immune response. Their isolation represents a major step in many immunological experiments. In this two phase study, we aimed to establish an optimum protocol for PBMC isolation by density-gradient centrifugation. Methods: During Phase-1, we compared two commercially available PBMC isolation protocols, Stemcell Technologies (ST) and Miltenyi Biotec (MB), in terms of PBMC recovery and purity. Twelve blood samples were assigned to each protocol. Each sample was divided in three subsamples of $1 \mathrm{ml}, 2 \mathrm{ml}$ and $3 \mathrm{ml}$ in order to assess the influence of blood sample volume on isolation performance. During Phase-2, a hybrid protocol was similarly tested, processing six blood samples. Additionally, we performed a flow cytometric analysis using an Annexin-V/Propidium-lodide viability staining protocol. Results: Phase-1 results showed that, for all subsample volumes, ST had superior PBMC recovery (mean values: $56 \%, 80 \%$ and $87 \%$, respectively) compared to MB (mean values: $39 \%, 54 \%$ and $43 \%$, respectively). However, platelet removal was significantly higher for MB (mean value of 96.8\%) than for ST (mean value of $75.2 \%$ ). Regarding granulocyte/erythrocyte contamination, both protocols performed similarly, yielding high purity PBMC (mean values: $97.3 \%$ for ST and 95.8\% for MB). During Phase-2, our hybrid protocol yielded comparable results to MB, with an average viability of $89.4 \%$ for lymphocytes and $16.9 \%$ for monocytes. Conclusions: ST yields higher cell recovery rates and MB excels at platelet removal, while the hybrid protocol is highly similar to $\mathrm{MB}$. Both cell recovery and viability increase with blood sample volume.
\end{abstract}

Keywords: density gradient centrifugation, PBMC isolation, PBMC viability

Received 29 May 2018 / Accepted 15 June 2018

\section{Introduction}

\section{Peripheral blood mononuclear cells (PBMC)}

Peripheral blood mononuclear cells (PBMC) are extremely important in the body's immune response. PBMCs are comprised of lymphocytes, monocytes and dendritic cells. In humans, lymphocytes account for most of the PBMCs, followed by monocytes and only a small fraction of dendritic cells. Given their involvement in virtually any immune process, PBMC isolation is a key step in many immunological experiments. Once isolated, PBMCs can be used for a wide range of downstream applications such as studying autoimmune diseases [1-3], cancer research [410], developing new vaccines [11-13] and immunotherapies [6-10], drug discovery and testing [14-16] etc.

Due to their practical importance in the study of the functional immune system, PBMCs represent an area of continuous interest. Over time, several descriptive and comparative studies have approached the issue of PBMC preparation by studying isolation principles $[17,18]$ and techniques, various isolation devices [18-23] and the effect of physical factors such as time [23-25], storage temperature [24, 26] and cryopreservation [22, 25, 27-29]. Commonly investigated parameters and performance indicators include cell recovery, cellular population composition, pu-

* Correspondence to: Doina Ramona Manu

E-mail: doina.manu@umftgm.ro rity, viability, sterility, activation status and functionality. So far, not a single isolation technique proved to be superior in all aspects. In fact, it is the researcher's responsibility to choose the most suitable method that fits a project's design, financial resources and available laboratory equipment and personnel.

One of the most commonly used techniques for PBMC isolation is the density gradient centrifugation method. The separation principle takes advantage of the slight difference in density among blood cells. Briefly, whole blood is first diluted with phosphate buffer saline (PBS) and then carefully layered over the density gradient medium. Granulocytes and erythrocytes have a higher density than mononuclear cells and therefore sediment through the bottom layer during centrifugation. PBMCs form a distinct band at the medium-plasma interface and will be carefully retrieved by pipette. Additionally, the freshly isolated cells can be resuspended in PBS for further centrifugation, which will subsequently increase purity.

\section{Aims and objectives}

In this two phase study, our aim was to establish an optimum protocol for PBMC isolation by density gradient centrifugation. If successful, the protocol would then be implemented as a standard operating procedure (SOP) at the Cellular Immunology Laboratory of the Center for Advanced Medical and Pharmaceutical Research (CCAMF) of the University of Medicine and Pharmacy of Tîrgu 
Mureş (UMFTGM, Romania). During Phase 1, two commercially available protocols - Stemcell Technologies (ST) and Miltenyi Biotec (MB) - were compared in terms of PBMC recovery and purity. Although based on the same density gradient principle, the two protocols recommend different ratios between blood, PBS and gradient medium. Moreover, the specified centrifugal force (CF, i.e. $\mathrm{xg}$ ) and centrifugation time (CT) as well as the number of cell washing steps are different. The schematic outline of Phase 1 and technical specifications are shown in Figure 1 and Table I, respectively. The symmetrical branched design of this phase is of great importance as it allows us to 1) compare performances between the two protocols; 2) assess the effect of blood sample volume on separation performance; 3) analyze the intraindividual variability for each of the 2 examiners; and 4) analyze the interindividual variability by comparing all corresponding results between the 2 examiners. During Phase 2, a hybrid protocol (HY) was tested for the same performance indicators to which we added a flow citometry assessment of viability.

Up to the present, no study has compared two density gradient protocols, namely Stemcell Technologies and Miltenyi Biotec. The present report addresses this gap and, to our knowledge, it is the first comparative analysis aiming to unravel the effect of CF, CT and number of washing steps on PBMC isolation performances.

\section{Methods}

\section{Samples and study design}

The study was approved by the UMFTGM Ethics Committee for Scientific Research (no.84/02.05.2017). The blood samples were collected from 12 healthy students who previously signed a written informed consent. The same student may have donated blood on multiple occasions.

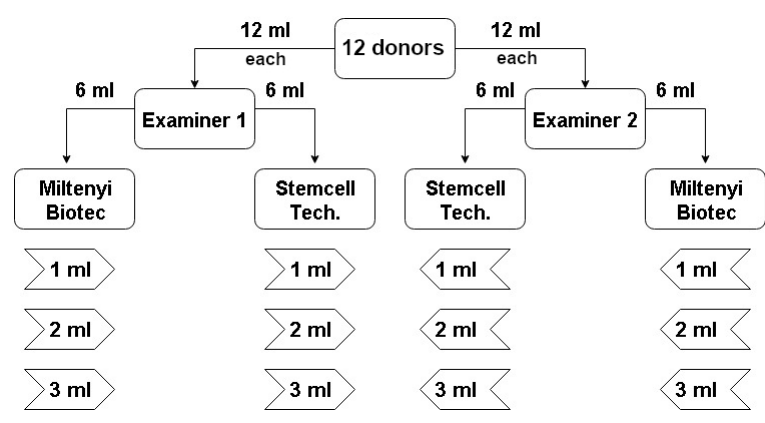

Fig. 1. Schematic outline of Phase-1. The symmetrical multibranched design allows for assessment of intra- and interindividual variability for different sample volumes
About $18 \mathrm{ml}$ of blood was collected from each donor using two $9 \mathrm{ml} \mathrm{NH}$ Sodium Heparin vacutainer tubes (Greiner Bio-One, catalog No.455051) from which the blood was processed within 1 hour and PBMC were separated within a maximum of 4 hours. The study was designed as a two phase laboratory experiment.

During Phase-1 of the study (see Figure 1), ST and MB were compared in terms of PBMC recovery and purity. Three distinct subsamples of blood $(1 \mathrm{ml}, 2 \mathrm{ml}$ and $3 \mathrm{ml})$ were assigned to each protocol in order to assess how sample volume influences the outcome of PBMC isolation. Furthermore, all 12 samples were processed by the same two examiners, 6 samples each, in a mirror-like manner as to cast light upon the effect of both intra- and interindividual variability on PBMC separation.

Based on the results from Phase-1, a new hybrid PBMC separation protocol was established and implemented in Phase- 2 where another 6 samples were processed by only one examiner. Throughout the study, PBMC recovery (\%), PBMC purity (\%) and Platelet removal (\%) were regarded as performance indicators. Additionally, a viability flow cytometric analysis was performed on all samples from Phase-2, using the FITC-conjugated early apoptosis marker Annexin-V and the late-apoptosis/necrosis marker Propidium Iodide (PI). Technical specifications of each protocol are detailed in Table I.

\section{Phase 1 - Stemcell Technologies vs. Miltenyi Biotec}

First, an automated complete blood count (CBC) was performed from each donor's blood sample using a SYSMEX XS-800i hematology analyzer. Each examiner was given $12 \mathrm{ml}$ of peripheral blood from their corresponding donor, $6 \mathrm{ml}$ to be processed according to ST and the other $6 \mathrm{ml}$ according to MB.

For ST, $6 \mathrm{ml}$ of blood was diluted (1:1) with Dulbecco's Phosphate Buffered Saline (DPBS; EuroClone, cata$\log$ No.ECB4053). Corresponding to a ratio of 2:1, volumes of $2 \mathrm{ml}, 4 \mathrm{ml}$ and $6 \mathrm{ml}$ from the diluted blood were carefully layered over $1 \mathrm{ml}, 2 \mathrm{ml}$ and $3 \mathrm{ml}$, respectively, of Histopaque-1077 (Sigma-Aldrich, catalog No.10771) in 3 distinct $15 \mathrm{ml}$ conical centrifuge tubes (VWR, catalog No.89039-666). The gradients were centrifuged at $800 \mathrm{xg}$ for $20 \mathrm{~min}$ at room temperature (RT), without brake. The newly formed PBMC bands were removed using fine tip pipettes (Thermo Scientific Samco, catalog No.22-610178 ) and transferred to 3 new $15 \mathrm{ml}$ tubes. The cells were then resuspended in DPBS up to a total volume of $14 \mathrm{ml}$ and centrifuged at $250 \mathrm{xg}$ for $10 \mathrm{~min}$ at RT, without brake.

Table I. Technical specifications for each protocol

\begin{tabular}{lcccccccc}
\hline & \multicolumn{3}{c}{ 1st step } & \multicolumn{2}{c}{ 2nd step } & 3rd step & 4th step \\
\cline { 2 - 7 } & B:PBS:H & CT & CF & CT & CF & CT & \\
\hline ST & $1: 1: 1$ & 20 & 800 & 10 & 250 & 10 & 200 \\
\hline MB & $1: 3: 1.7$ & 30 & 400 & 10 & 300 & MB & bis \\
\hline HY & ST & & & & & Viability \\
\hline
\end{tabular}

P:PBS:H - blood:PBS:Histopaque ratio; CT - centrifugation time $(\mathrm{min})$; CF - centrifugal force $(\mathrm{xg})$ 
To simplify calculations, after discarding the supernatant, pellets were resuspended in DPBS up to the original volume of blood that was processed for each. Finally, a CBC was performed from each tube in order to determine the total number of cells and PBMC populations, while performance indicators were computed with respect to the initial whole blood CBC.

For $\mathrm{MB}, 6 \mathrm{ml}$ of blood was diluted (1:3) with DPBS. Corresponding to a ratio of $7: 3$, volumes of $4 \mathrm{ml}$ and $8 \mathrm{ml}$ from the diluted blood were carefully layered over $1.7 \mathrm{ml}$ and $3.4 \mathrm{ml}$, respectively, of Histopaque- 1077 in 2 distinct $15 \mathrm{ml}$ centrifuge tubes. The remaining $12 \mathrm{ml}$ of diluted blood was similarly layered over $5.1 \mathrm{ml}$ of Histopaque-1077, in a $50 \mathrm{ml}$ centrifuge tube (VWR, catalog No.89039-656). The gradients were centrifuged at 400xg for $30 \mathrm{~min}$ at RT, without brake. The newly formed PBMC bands were removed using fine tip pipettes and transferred to 3 new $15 \mathrm{ml}$ tubes. The cells were then resuspended in DPBS up to the volume of $14 \mathrm{ml}$ and centrifuged at $300 \mathrm{xg}$ for $10 \mathrm{~min}$ at RT, without brake. After discarding the supernatant, pellets were similarly washed two more times and centrifuged at $200 \mathrm{xg}$ for $10 \mathrm{~min}$. As in the first phase, pellets were resuspended in DPBS up to the original volume of blood that was processed for each and a $\mathrm{CBC}$ was performed from each tube in order to calculate the performance indicators.

\section{Phase 2 - Hybrid protocol and Viability}

Following interpretation of data from Phase-1, a likely more effective hybrid (HY) PBMC isolation protocol was proposed. A series of 6 PBMC separations was performed by a single examiner using the same equipment mentioned above. After performing an initial CBC, $6 \mathrm{ml}$ of blood were processed following the complete ST proto$\mathrm{col}$, as previously described. Additionally, to reduce platelet contamination, pellets from all three tubes were washed two more times according to MB protocol $(200 \mathrm{xg}, 10 \mathrm{~min}$, $\mathrm{RT}$, without brake). After performing a final CBC from each tube, the samples were washed with DPBS and centrifuged at $400 \mathrm{xg}$ for $10 \mathrm{~min}$ at RT without brake and the supernatants were discarded. For the viability staining protocol, a FITC-Annexin-V Apoptosis Detection Kit I (BD Pharmigen, catalog No.556547) was used. Based on the final CBCs, calculations were made and pellets were resuspended in Binding Buffer at a concentration of $1 \times 10^{6}$ cells/ $\mathrm{ml}$. From each tube, $100 \mu \mathrm{L}$ of solution $\left(1 \times 10^{5}\right.$ cells $)$ was transferred to distinct $5 \mathrm{ml}$ tubes. FITC-Annexin-V and PI, $5 \mu \mathrm{l}$ of each, were added to all tubes and the cells were incubated for $15 \mathrm{~min}$ at RT in the dark. After the incubation, $400 \mu \mathrm{l}$ of Binding Buffer was added to each tube and cells were analyzed within 30 minutes using FACSAria III flow cytometer with FACSDiva v8.0 Software (both BectonDickinson Biosciences). Based on cell morphology (FSC/ SSC), PBMCs were gated into two main populations lymphocytes and monocytes - and a secondary population including all other non-debris events (Figure 2a). The vi- ability assessment (FITC/PI) was performed only on gated singlets (Figure 2b).

\section{Statistical processing}

In our study, the significance threshold was set at 0.05 . All statistical processing was performed using Microsoft Excel, GraphPad Prism 6 or BD FacsDiva-v8.0. A KolmogorovSmirnov normality test was applied for each data set. Intraindividual analysis between data sets of different sample volumes was performed using the paired $\mathrm{t}$-Test, while for interindividual analysis, the two-sample t-Test was applied. All significant differences were reported using two-tailed p-values.

\section{Results}

All numerical values from Phase- 1 are presented in Table II. Each examiner's sets of values have been statistically analyzed and found to be normally distributed. No intraindividual variability was identified $(p>0.05)$, regardless of what examiner/protocol/volume association was considered (data not shown).

A quick analysis of Table II reveals highly similar values between E1 and E2 which suggests low interindividual variability, also confirmed by stastical analysis $(p>0.05)$. One exception is the MB- $3 \mathrm{ml}$ lot where PBMC recovery is significantly higher for $\mathrm{E} 1(\mathrm{p}=0.005)$. Given the observed low interindividual variability, an average between E1 and E2 was calculated and the results were also presented in Table II. Although (\%) is a better performance indicator, we have also calculated the PBMC recovery in "million cells per $m l$ of blood" which is a more practical manner of reporting cell recovery. Average PBMC recovery values for ST’s $1 \mathrm{ml}, 2 \mathrm{ml}$ and $3 \mathrm{ml}$ sample lots are 1.44, 2.04 and 2.25 million cells per $\mathrm{ml}$ of blood, respectively. For $\mathrm{MB}$, the corresponding values are 1.17, 1.59 and 1.28 million cells per ml of blood, respectively. It is also obvious that higher sample volumes yielded higher recovery rates, an observation that was also supported by statistical analysis ( $\mathrm{p}$ values not shown), the only exception being again the MB-3ml lot. PLT removal was calculated by comparing the initial and final CBCs, using the following formula: PLT removal $=($ initial PLT - final PLT)/initial PLT. By comparing ST and MB in terms of PLT removal, it is evident that MB has an unquestionably higher PLT removal rate $(\mathrm{p}<0.0001$ for all three sample volumes), with an average of $6 x$ less contaminant PLTs for MB samples than for ST. For MB, there are no differences between blood sample lots regarding PLT contamination while for ST, the $1 \mathrm{ml}$ lot was significantly less contaminated than its $2 \mathrm{ml}$ and $3 \mathrm{ml}$ counterparts $(\mathrm{p}=0.004$ and $\mathrm{p}=0.0006$, respectively). It should be specified that, for both protocols, erythrocyte contamination was very low or absent (data not shown), while granulocyte contamination is represented by the "PBMC purity" performance indicator described in Table II. In general, contaminating granulocytes were mainly comprised of neutrophils with scarce eosinophils and basophils (data now shown). Regarding 

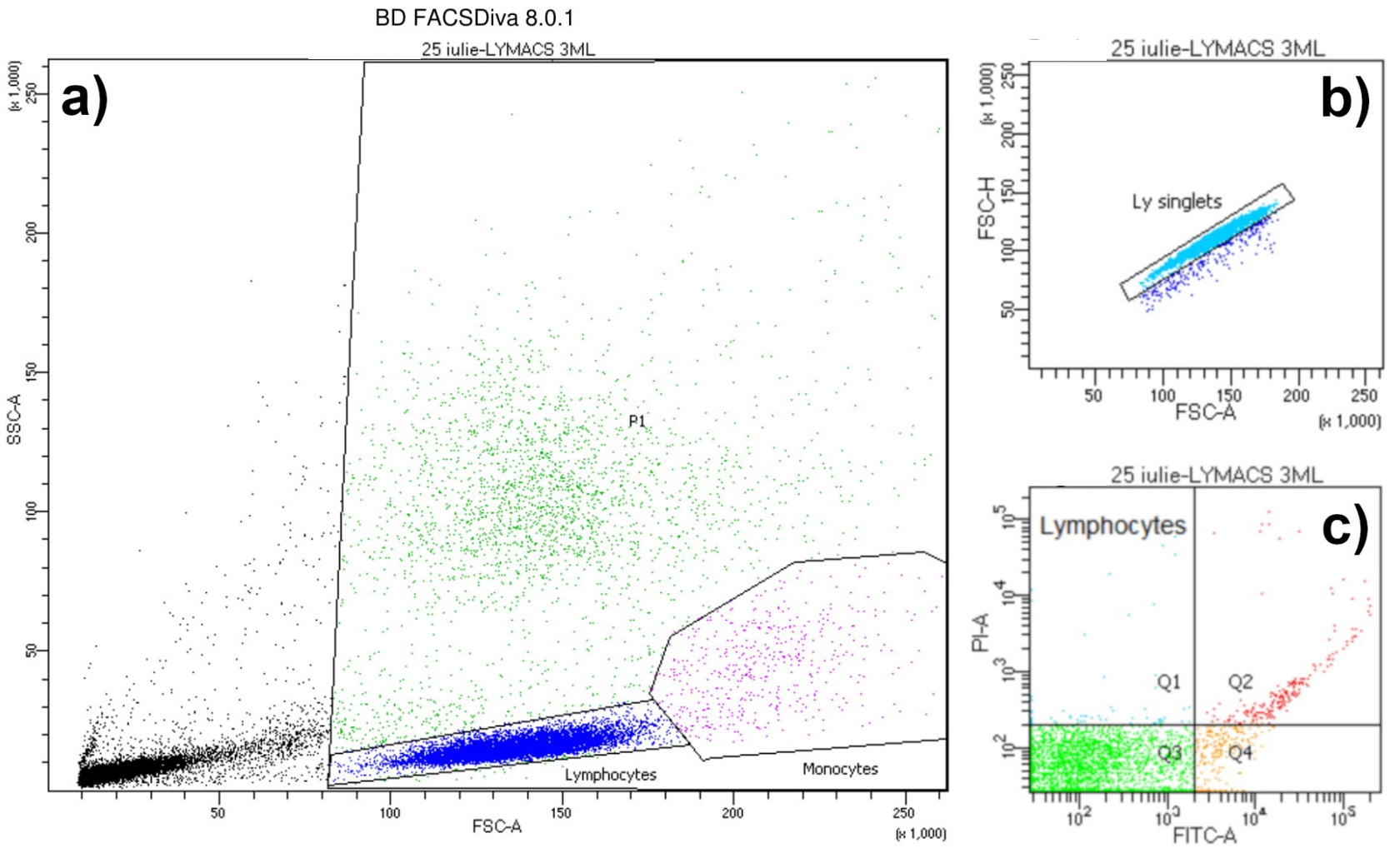

Fig. 2. An illustrative partial flow cytometry report showing a) gating of PBMCs into distinct populations based on morphological parameters (FSC/SSC); b) doublet discrimination and c) assessment of viability by FITC-Annexin-V and PI staining positivity

Table II. Average numerical values from Phase-1

\begin{tabular}{|c|c|c|c|c|c|c|c|c|c|c|}
\hline \multirow{2}{*}{\multicolumn{2}{|c|}{$1 \mathrm{ml}$}} & \multicolumn{3}{|c|}{ PBMC recovery (\%) } & \multicolumn{3}{|c|}{ PLT removal (\%) } & \multicolumn{3}{|c|}{ PBMC purity* (\%) } \\
\hline & & $2 \mathrm{ml}$ & $3 \mathrm{ml}$ & $1 \mathrm{ml}$ & $2 \mathrm{ml}$ & $3 \mathrm{ml}$ & $1 \mathrm{ml}$ & $2 \mathrm{ml}$ & $3 \mathrm{ml}$ & \\
\hline \multirow{3}{*}{ ST } & E1 & 55.8 & 79.7 & 85.9 & 81.1 & 76.0 & 72.8 & 95.1 & 98.6 & 97.4 \\
\hline & E2 & 55.5 & 79.7 & 87.9 & 83.5 & 70.6 & 67.4 & 98.0 & 98.2 & 96.4 \\
\hline & Avg. & 55.7 & 79.7 & 86.9 & 82.3 & 73.3 & 70.1 & 96.6 & 98.4 & 96.9 \\
\hline \multirow{3}{*}{ MB } & E1 & 40.3 & 52.8 & 51.8 & 96.7 & 97.2 & 97.1 & 97.2 & 97.5 & 98.3 \\
\hline & E2 & 37.5 & 55.2 & 34.2 & 96.8 & 96.3 & 96.7 & 93.1 & 94.7 & 94.0 \\
\hline & Avg. & 38.9 & 54.0 & 43.0 & 96.7 & 96.8 & 96.9 & 95.2 & 96.1 & 96.2 \\
\hline
\end{tabular}

cell population composition, both examiners had similar results, independent of protocol and blood sample volume. The average PBMC population composition throughout Phase- 1 is $86.4 \%$ lymphocytes and $10.2 \%$ monocytes (data not shown).

For Phase-2, all numerical values are presented in Table III. As expected, PBMC recovery significantly increases with sample volume ( $\mathrm{p}=0.002$ for $2 \mathrm{ml}$ vs. $1 \mathrm{ml} ; \mathrm{p}=0.04$ for $3 \mathrm{ml}$ vs. $2 \mathrm{ml}$ ). Average cell recovery values reported in million cells per $\mathrm{ml}$ of blood are as follows: 1.00 for $1 \mathrm{ml}, 1.57$ for $2 \mathrm{ml}$ and 1.81 for $3 \mathrm{ml}$. It should be stressed that PBMC recovery, PLT removal and purity values are very similar between HY and MB protocols (see Tables II and III). PLT removal was significantly greater for the $1 \mathrm{ml}$ lot than for its $2 \mathrm{ml}(\mathrm{p}=0.006)$ and $3 \mathrm{ml}(\mathrm{p}=0.04)$ counterparts. As shown in Figure 2c, in this study, cells considered viable are FITC Annexin V and PI negative; cells in early apoptosis are FITC Annexin V positive and PI negative; and cells in late apoptosis or already dead are both FITC Annexin V and PI positive. Lymphocyte viability was generally high and also significantly increases with sample volume $(\mathrm{p}=0.001$ for $2 \mathrm{ml}$ vs. $1 \mathrm{ml} ; \mathrm{p}=0.02$ for $3 \mathrm{ml}$ vs. $2 \mathrm{ml}$ ). On the contrary, we recorded low viabilities for monocytes, while no influence of sample volume was observed $(\mathrm{p}>0.05)$. Although increasing with sample volume, the viability of non-lymphocyte-non-monocyte events was also low with only the $3 \mathrm{ml}$ lot showing significantly higher viability than the $1 \mathrm{ml}$ and $2 \mathrm{ml}$ lots ( $\mathrm{p}=0.04$ and $\mathrm{p}=0.008$, respectively).

\section{Discussion}

\section{Density gradient centrifugation - advantages and limi- tations}

There are several ways of isolating PBMCs, each technique having its own advantages and limitations [17,18]. The density gradient separation principle was first described by B̈̈yum A. [30, 31] and quickly became the most popular PBMC preparation method. A major advantage of this method for lymphocyte immunophenotyping is the removal of most erythrocytes, granulocytes and nonvi- 
Table III. Average numerical values from Phase-2

\begin{tabular}{|c|c|c|c|c|}
\hline & & $1 \mathrm{ml}$ & $2 \mathrm{ml}$ & $3 \mathrm{ml}$ \\
\hline \multicolumn{2}{|c|}{ PBMC recovery (\%) } & 37.2 & 57.3 & 63.7 \\
\hline \multicolumn{2}{|c|}{ PLT removal (\%) } & 98.1 & 95.5 & 93.9 \\
\hline \multicolumn{2}{|c|}{ PBMC purity (\%) } & 97.0 & 95.4 & 92.6 \\
\hline \multirow{3}{*}{ Viability (\%) } & Lymphocytes & 86.2 & 90.2 & 91.7 \\
\hline & Monocytes & 16.1 & 17.0 & 17.5 \\
\hline & Other* & 25.7 & 26.2 & 33.1 \\
\hline
\end{tabular}

able cells from the sample [17]. Therefore, initial purification of cell populations speeds up the acquisition process while saving expensive antibodies by reducing non-specific binding. Density centrifugation is the most typical density gradient method to isolate PBMCs. Despite the advent of other enhanced, easier-to-use techniques, density centrifugation reportedly remains the most employed density-based PBMC isolation method. Besides yielding good results, this method is also less expensive [19] and less complicated than many of the other methods, allowing for relatively rapid processing with minimal laboratory equipment requirements. The main limitation of our study was the lack of population-specific staining markers such as CD3, CD4, CD8 for lymphocytes and CD14 for monocytes. Although morphology-based gating (FSC/ SSC) allows for good population identification, it gives no information about lymphocyte sub-populations or any of the non-debris gated events that were too spread out to be accurately identified.

There are two critical steps in PBMC separation by density centrifugation. The first one is layering blood over the gradient medium which is a delicate process where patience and self control prevent catastrophic mixing of the two layers. At the end of this step, one should clearly see a distinct separation between the clear bottom layer and the upper blood layer. Inadequate layering during this step may result in partial or complete loss of the target cells. The second critical step is extracting the buffy coat which is found at the medium-plasma interface. Contamination is inevitable but should be kept minimal as the osmotic stress exerted by the gradient medium may result in decreased viability, while the upper plasma layer is a rich source of undesired platelets.

\section{PBMC recovery, purity, viability and platelet contami- nation}

This study showed that $\mathrm{ST}$ protocol is superior to $\mathrm{MB}$ in terms of PBMC recovery (Figure $3 \mathrm{a}$ vs. $3 \mathrm{~b}$ ), while MB excels in platelet removal, with significantly lower platelet contamination (Figure 3c). For MB, there are no differences between blood sample lots regarding platelet contamination while for ST, the $1 \mathrm{ml}$ lot was significantly less contaminated than its $2 \mathrm{ml}$ and $3 \mathrm{ml}$ counterparts. Regarding cell recovery, our results are consistent with previous studies reporting an average cell recovery by Ficoll isolation between 0.6 and 3 million cells per $\mathrm{ml}$ of blood [19-22]. However, we consider that when assessing performance, "cells per ml of blood" is an inappropriate manner of reporting cell recovery since it provides no information about initial cell count and cell population composition. Both protocols showed high and comparable PBMC purities, with sporadic contamination due to residual erythrocytes and granulocytes. HY protocol consisted of a complete ST protocol to which we added the last two washing steps from MB. All performance indicators were similar between $\mathrm{MB}$ and HY protocols (compare Tables II and III, see Figure $3 \mathrm{~d}, \mathrm{e})$. Therefore, the lower cell recovery and PLT contamination observed in MB/HY can only be explained by the two additional washing steps that were performed for these protocols but not for ST. Monocytes are well known for their fragility in such experiments, while lymphocytes are typically more resistant, hence the generally highlymphocyte/low-monocyte viabilities seen in our hybrid protocol (Figure 3f). We would like to emphasize the ascending trend seen in Phase-2, where lymphocyte viabil- a)

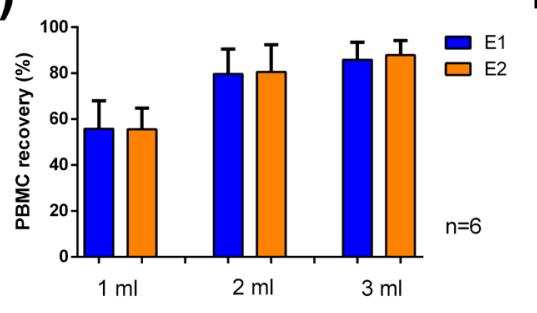

b)

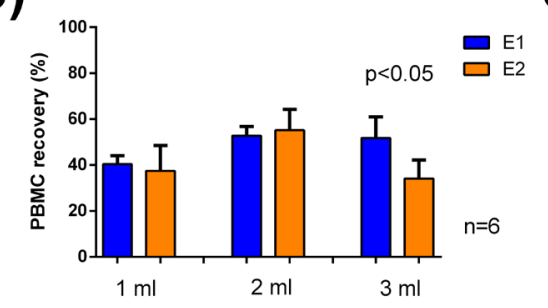

c)

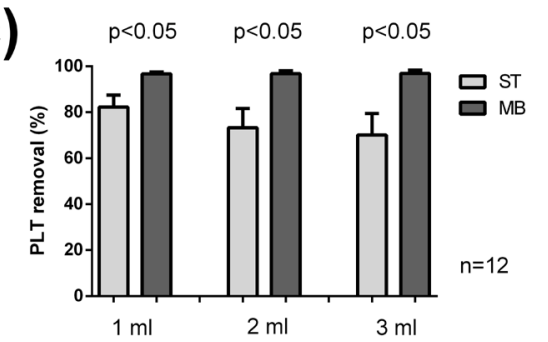

d)

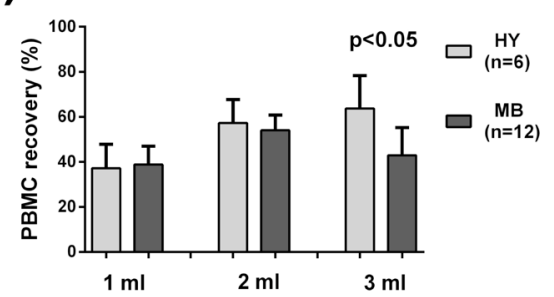

e)

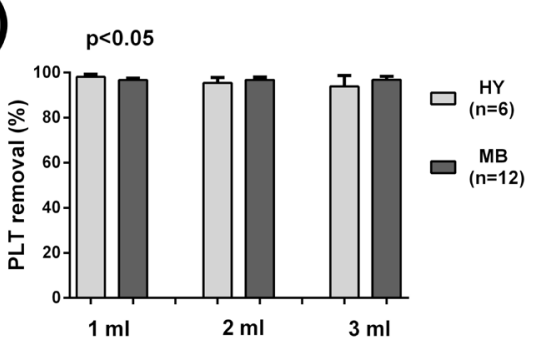

f)

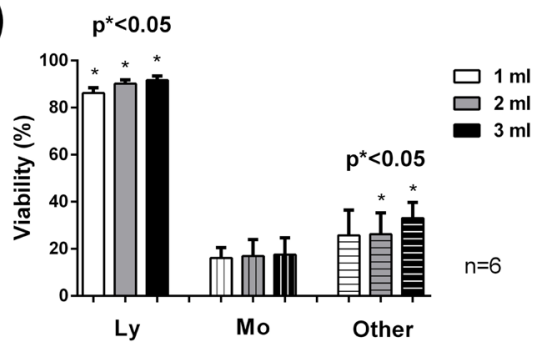

Fig. 3. Column bar graph representing average variation of recovery rates with sample volume for a) ST; b) MB; d) HY. Average platelet removal is compared between MB and c) ST and e) HY. Sub-figure e) shows average viability for cell populations as 
ity significantly increases with blood sample volume. HY protocol's resemblance to $\mathrm{MB}$ in both design and outcome supports the idea that, if tested for viability, MB would yield similar results. Due to its single washing step, ST protocol may have higher levels of residual Histopaque-1077 which can cause cellular toxicity. On the other hand, ST may yield a higher viability since this protocol is notably faster and requires less centrifugation and cell manipulation than HY/MB. It's also worth mentioning that in our experiment, cells were resuspended and washed in PBS at RT while complex downstream applications require that washing steps be done with culture media. In this case, an increase in viability should be expected.

\section{Blood sample volumes and variability}

An important aspect of our study was the intra- and interindividual variability of the two examiners. Each of them processed a total of 36 samples that is 18 samples per protocol. For each protocol, the 18 samples were divided in 3 lots of 6 , namely $1 \mathrm{ml}, 2 \mathrm{ml}$ and $3 \mathrm{ml}$. Regardless of which protocol and/or examiner was considered, there were no relevant differences between cell recovery rates within any of the lots, meaning that both examiners showed insignificant intraindividual variability. Regarding the interindividual variability, it has been previously reported that differences up to $60 \%$ in cell recovery were recorded between a well-trained but inexperienced technician and a technician experienced in cell isolations [19]. In our experiment, the examiners were similarly trained/experienced and processed the samples at the same time in identical conditions. When the corresponding lots were compared between the two, we found no considerable differences in cell recovery, except for the $3 \mathrm{ml}$ lot from MB protocol (Figure 3a,b). The most likely cause of this disparity is that $\mathrm{MB}-3 \mathrm{ml}$ was the only lot where samples were processed in (2/3 empty) $50 \mathrm{ml}$ centrifuge tubes, thus making the buffy coat removal an even more challenging task. Nonetheless, we conclude that in our experiment, the degree of interindividual variability is negligible.

Another important aspect is the effect of blood sample volume on separation performance. We have chosen to investigate blood volumes of $1 \mathrm{ml}, 2 \mathrm{ml}$ and $3 \mathrm{ml}$ as such small volumes are likely obtainable in most clinical contexts. Moreover, assuming the donor's CBC is within normal range, even a $50 \%$ recovery rate should deliver roughly between 0.5 and 2.0 million PBMCs $/ \mathrm{ml}$ of blood, which is enough for immunophenotyping purposes and other preliminary analyses and processing. In our study, regardless of the employed protocol, both examiners recorded the same ascending trend where cell recovery rates increased with blood sample volume (Figure 3a,b). This trend could also be observed in Phase-2 with the hybrid protocol for both cell recovery and viability (Figure 3d,f), but the extent and limitations of this effect remain to be further investigated.

\section{Practical aspects}

The low interindividual variability recorded in our experiment allowed us to cumulate the results from both examiners and perform a global and statistically more powerful analysis that further consolidated our conclusions. However, a series of aspects needs to be taken in consideration. Given that $\mathrm{MB}$ is considerably more expensive and time consuming than ST protocol, routinely employing MB for $\mathrm{PBMC}$ isolation is questionable unless low platelet contamination is required. In our study, the two examiners were similarly experienced/trained, hence the minimal intra- and interindividual variability. However, this is not always the case, especially with large studies employing both experienced and newly recruited personnel. Therefore, we stress that variability should not be overlooked. It was clearly shown that blood sample volume influences separation performance. Although some volumes proved superior to others, it is sometimes physically impossible or ethically questionable to collect the desired amount of blood. Also, any study involving PBMC isolation as a first step should take in consideration whether granulocytes and PLTs can at any point interfere and influence the results. Consequently, the most appropriate protocol should be chosen. Another question is when to stop washing the cells. It is obvious that additional wash cycles considerably reduce PLT contamination, but the absolute PBMC count also decreases with each cycle. Therefore, a convenient compromise should be reached between cell recovery and platelet contamination. Last but not least, obtaining viable cells is the main objective of most experiments involving PBMCs. During separation, PBMCs are exposed to chemical, mechanical and osmotic stress, hence the importance of minimal cell manipulation techniques and appropriate buffers and culture media. If satisfactory, the shortest and less stressful protocol should be employed as each minute of in vitro processing takes its toll on viability. We have already described the two critical steps of PBMC isolation by density gradient centrifugation and we suggest that individuals using this technique should be at least averagely trained and experienced in order to achieve satisfactory results.

A set of general indications was formulated to serve as a guideline for contextual adjustment of density gradient centrifugation protocols for PBMC isolation:

1. If kept within normal range, $\mathrm{CF}, \mathrm{CT}$ and Blood: PBS: gradient medium ratio can be varied

2. MB protocol should not be employed on a routine basis unless otherwise specified

3. For maximum PBMC recovery, ST/ST-like protocol is recommended; if possible, use blood samples of $3 \mathrm{ml}$ in order to obtain a total amount of 1.8-12.0 million PBMCs (computed for the worst/best-case scenarios of a physiological CBC)

4. For minimal PLT contamination, a multi-wash-ST/ HY protocol is recommended

5. For maximum viability, use samples of $3 \mathrm{ml}$ 
Following this study, ST and HY protocols were successfully tested and implemented as SOPs in the CCAMF Cellular Immunology Laboratory. However, for future studies, these protocols may be applied for both healthy and pathological subjects. Selection of 'healthy' control subjects will require preliminary basic screening and subsequent exclusion of inflammatory status and/or abnormal CBCs. Depending on clinical context and each study's design, aims and particularities, pathological subjects may be either excluded or required to donate an increased amount of blood in order to obtain the desired number of target cells (e.g. leukopenia, abnormal leukocyte subpopulations distribution). However, further investigations are required in order to properly establish the applicability of ST/HY protocols for this particular cases.

\section{Conclusions}

Overall, this study provided us with a valuable insight on how density centrifugation separation protocols could be adjusted in order to meet the performance criteria required by various downstream applications. We confirm that both ST and MB are reliable protocols, yielding satisfactory cell recovery rates and purities. ST is considerably faster and cheaper than $\mathrm{MB}$ and also yields more cells while $\mathrm{MB}$ stands out for its exceptionally low PLT contamination. Multiple washing steps decrease both cell recovery and PLT contamination. The hybrid protocol is as good and solid as MB but less expensive and shows acceptable lymphocyte viability despite its many steps and long duration. Regarding sample volumes, we conclude that, up to at least $3 \mathrm{ml}$, the larger the blood sample, the higher the cell recovery rates will be. Intra- and interindividual variability should not be an issue if the examiners are equally trained and experienced. Another conclusion is that differences in Blood: PBS: Ficoll ratio, CF and CT between ST and MB/ HY may have little to no effect on cell recovery and erythrocyte/granulocyte contamination.

\section{Conflict of interest}

None to declare.

\section{Acknowledgements}

This work was partially supported by a grant of the Romanian National Authority for Scientific Research and Innovation CNCS/UEFISCDI, [PN-III-P2-2.1PED-2016-0734], contract no.155 PED/2017.

\section{References}

1. Mesko B., Poliska S., Nagy L.- Gene expression profiles in peripheral blood for the diagnosis of autoimmune diseases. Trends Mol Med 2011;17(4):223-233.

2. Olsen N.J., Moore J.H., Aune T.M. - Gene expression signatures for autoimmune disease in peripheral blood mononuclear cells. Arthritis Res Ther 2004;6(3):120-128.

3. ShiG., Zhang Z., Li Q. - New Biomarkers in Autoimmune Disease. Hindawi, J Immunol Res 2017;https://doi.org/10.1155/2017/8702425.

4. Harouaka R., Kang Z., Zheng S. et al. - Circulating tumor cells: advances in isolation and analysis, and challenges for clinical applications.
Pharmacol Ther. 2014;141(2):209-221.

5. Esmaeilsabzali H., Beischlag T.V., Cox M.E. et al. - Detection and isolation of circulating tumor cells: Principles and methods. Biotechnol Adv, 2013;31(7):1063-1084.

6. Masucci G.V., Cesano A., Hawtin R. et al. - Validation of biomarkers to predict response to immunotherapy in cancer: Volume I - pre-analytical and analytical validation. J Immunother Cancer 2016;4:76.

7. Farkona S., Diamandis E.P., Blasutig I.M.- Cancer immunotherapy: the beginning of the end of cancer?. BMC Med. 2016;14:73.

8. Vile R.G.- Socializing Individualized T-Cell Cancer Immunotherapy.

9. Mol. Ther 2016;24(7):1170-1173.

10. Yuan J., Hegde P.S., Clynes R. et al. - Novel technologies and emerging biomarkers for personalized cancer immunotherapy. J Immunother Cancer 2016;4:3.

11. Kohrt H.E., Tumeh P.C., Benson D. et al. - Immunodynamics: a cancer immunotherapy trials network review of immune monitoring in immunooncology clinical trials. J Immunother Cancer 2016;4:15.

12. Kumar S., Sunagar R., Pham G. et al. - Ex vivo antigen-pulsed PBMCs generate potent and long lasting immunity to infection when administered as a vaccine. Vaccine 2017;35(7):1080-1086.

13. Sambor A., Garcia A., Berrong M.et al. - Establishment and maintenance of a PBMC repository for functional cellular studies in support of clinical vaccine trials. J Immunol Methods 2014;0:107-116.

14. Bürdek M., Spranger S., Wilde S.et al. - Three-day dendritic cells for vaccine development: Antigen uptake, processing and presentation. J Transl Med 2010;8:90.

15. Frijters R., van Vugt M., Smeets R.et al. - Literature Mining for the Discovery of Hidden Connections between Drugs, Genes and Diseases. PLoS Comput Biol 2010;6(9):e1000943.

16. Heath J.R., Ribas A., Mischel P.S.- Single cell analytic tools for drug discovery and development. Nat Rev Drug Discov. 2016;15(3):204-216.

17. Pourahmad J., Salimi A. - Isolated Human Peripheral Blood Mononuclear Cell (PBMC), a Cost Effective Tool for Predicting Immunosuppressive Effects of Drugs and Xenobiotics. Iran J Pharm Res 2015;14(4):679980.

18. Dagur P.K., McCoy Jr. J.P. - Collection, Storage, and Preparation of Human Blood Cells. Curr Protoc Cytom. 2016;73:5.1.1-5.1.16.

19. Rahmanian N., Bozorgmehr M., Torabi M. et al. - Cell separation: Potentials and pitfalls. Prep Biochem Biotechnol 2017;47(1):38-51.

20. Grievink H.W., Luisman T., Kluft C. et al. - Comparison of Three Isolation Techniques for Human Peripheral Blood Mononuclear Cells: Cell Recovery and Viability, Population Composition, and Cell Functionality. Biopreserv Biobank 2016;14(5):410-415.

21. Stone M., Murcia K., Dimapasoc M. et al. - Maximizing PMBC Recovery and Viability. A Method to Optimize and Streamline Peripheral Blood Mononuclear Cell Isolation, Cryopreservation, and Thawing. Bioprocess Int 2015;13(4)s.

22. Nilsson C., Aboud S., Karle K. et al. - Optimal Blood Mononuclear Cell Isolation Procedures for Gamma Interferon Enzyme-Linked Immunospot Testing of Healthy Swedish and Tanzanian Subjects. Clin. Vaccine Immunol. 2008;15(4):585-589.

23. Ruitenberg J.J., Mulder C.B., Maino V.C. et al. - VACUTAINER® CPT and Ficoll density gradient separationperform equivalently in maintaining the quality and function of PBMC from HIV seropositive blood samples. BMC Immunol. 2006;7(11).

24. Apithy M.-J., Desoutter J., Guillaume N.- Comparison of two peripheral mononuclear cell isolation protocols for flow cytometry crossmatching. doi: 10.1111/tan.13192.

25. Debey S., Schoenbeck U., Hellmich M. et al. - Comparison of different isolation techniques prior gene expression profiling of blood derived cells: impact on physiological responses, on overall expression and the role of different cell types. Pharmacogenomics J. 2004;4(3):193-207.

26. Palmirotta R., De Marchis M. L., Ludovici G. et al. - Impact of preanalytical handling and timing for peripheral blood mononuclear cells isolation and RNA studies: the experience of the Interinstitutional Multidisciplinary BioBank (BioBIM). Int. J. Biol. Markers 2012;27(2):90-98.

27. Berhanu D., Mortari F., De Rosa S.C. et al. - Optimized lymphocyte isolation methods for analysis of chemokine receptor expression. J Immunol Methods 2003;279(1-2):199 - 207.

28. Ramachandran H., Laux J., Moldovan I. et al. - Optimal Thawing of Cryopreserved Peripheral Blood Mononuclear Cells for Use in HighThroughput Human Immune Monitoring Studies. Cells 2012;1(3):313324.

29. Hønge B. L., Petersen M. S., Olesen R. et al. - Optimizing recovery of frozen human peripheral blood mononuclear cells for flow cytometry. PLOS ONE 2017;12(11):e0187440. 
30. Yang J., Diaz N., Adelsberger J. et al. - The effects of storage temperature on PBMC gene expression. BMC Immunol. 2016;17:6.

31. Boyum A. - Isolation of mononuclear cells and granulocytes from human blood. Scand. J. Clin. Lab. Invest. Suppl. 1968;97:77-89.

32. Boyum A. - Separation of lymphocytes, lymphocyte subgroups and monocytes: A review. Lymphology 1977;10(2):71-76. 